\title{
Bayesian Approach: An Alternative to Periodogram and Time Axes Estimation for Known and Unknown White Noise
}

\author{
Olanrewaju Rasaki Olawale a \\ ${ }^{a}$ Department of Statistics, University of Ibadan, Ibadan, 200284, Nigeria.
}

Received: 14 February 2018; Accepted: 08 March 2018; Published: 08 April 2018

\begin{abstract}
This study describes the Bayesian approach as an alternative approach for estimating time axes parameters and the periodogram (power spectrum) associated with sinusoidal model when the white noise (sigma) is known or unknown. The conventional method of estimating the time axes parameters and the periodogram has been via the Schuster method that relies solely on Maximum Likelihood Estimation (MLE). The Bayesian alternative approach proposed in this work, on the other hand, adopted the Maximum a Posteriori (MAP) via the Markov Chain Monte Carlo (MCMC) in order to checkmate the problem of re-parameterization and overparameterization associated with MLE in the conventional practice. The rates of heartbeat variability at exactly an hour and two hours after birth of one thousand eight hundred (1800) newly born babies in a state hospital were recorded and subjected to both the Bayesian approach and Schuster approach for inferences. The periodogram estimates, exactly an hour and two hours of after birth, were estimated to be 0.7395 and 0.7549 , respectively - and it was deduced that rates of heartbeat (frequency) variability moderated and stabilized the pulse among the babies after two hours of birth. In addition, MAP mean estimates of the parameters approximately equals to the true mean of estimates when round up to curb the problem of re-parameterization and over- parameterization that do affect Schuster method via MLE.
\end{abstract}

Index Terms: Bayesian, Maximum A Posteriori (MAP), Markov Chain Monte Carlo (MCMC), Maximum Likelihood Estimation (MLE), and Periodograms.

(C) 2018 Published by MECS Publisher. Selection and/or peer review under responsibility of the Research Association of Modern Education and Computer Science

\section{Introduction}

Time series modeling and data analysis are conventionally related to Bayesian data analysis with its general

* Corresponding author. Tel: 08060254814

E-mail address: rasakiolawale@gmail.com 
approach to modeling methods and its principles. It is a known, and already established fact that stochastic time series models evolve round deterministic (which is attributed to frequency change or Fourier decomposition in voice signals, vibrations, Electrocardiogram (ECG) etc.) time series for parameters embedded in sinusoidal model to be proper studied and interpreted. The typical and well-known frequency is the periodogram; according to [1], periodogram which is otherwise known as classical Fourier Power Spectrum is closely related to the Posterior Probability Density Function (PDF) function of a Bayesian setting over the frequency parameter of a Sinusoidal model. This implies that a Posterior PDF function $P(\theta / U, M)$ is needed for a given model " $M$ " with it values of parameters " $\theta$ " that best describes the data " $U$ ".

\section{Nomenclature}

\begin{tabular}{|ll|}
\hline$\omega$ & Periodogram \\
$\theta$ & Parameter vector or space \\
$A \& B$ & Time axes \\
$y$ & Single time series variable \\
$U$ & Set of events with variable of constant time varying variation \\
$M$ & Model \\
$\varepsilon\left(t_{i}\right)$ & White noise process \\
$\sigma$ & Noise (sigma) \\
$f(t)$ & Sinusoidal model \\
$p(\sigma)$ & Jeffrey's prior \\
$P(\omega, \sigma / U, M)$ Bayesian periodogram \\
\hline
\end{tabular}

\section{Related Work}

Contributions by [2] and [3] cannot be mentioned when it comes to the Singular Spectrum Analysis (SSA) approach of time axes via oscillating component of the unknown periodogram and the use of non-parametric prior approach on spectral density to established pseudo-posterior distribution for a short-memory Gaussian time series under some conditions on the prior for frequency time series model respectively. A well-provided method for calculating signaling time of the community model via late signaling cost for the data fusion using the Dynamic Transformation Model (DTM) by [4] has been the link between two processes in signaling and time axes indexes; the signaling time was estimated based on the data transmission time and processing delay based on the two immediate filter levels via designed algorithm.

[5] gave a clear picture of how spectral time series of multispectral and periodogram recognition schemes in the contexts of image acquisition, iris segmentation, texture analysis, and matching and performance evaluation while [6] thoroughly dealt with Fourier analysis on graphs with both positive and negative edges; [6] investigated the impacts of introducing negative edges and examine patterns in the spectral space of the graphs' adjacency matrix. Their theoretical results [5] and [6] showed that communities in a $k$-balanced signed graph are distinguishable in the spectral space of its signed adjacency matrix even if connections between communities are dense with an illustration empirical evaluation on both synthetic data and real life data. [7] Also maintained that the Wigner quasi-distribution plays an alternative role in both time-frequency analysis and quantum mechanics the white noise instead of the conventional Gaussian distribution been use in both the 
classical and suggested Bayesian approach. They all maintained the ground of estimating the periodogram and time axes parameters via the classical approach. This research gives an insight of estimating the parameters via Bayesian approach with or without the prior knowledge of the noise (sigma).

\subsection{Bayesian Analytical Approach in Estimating the Periodogram via the Sinusoidal Model}

Considering a single variable time series with variation (wave) of a single quantity, " $y$ " with time " $t$ " in a set of events $U=\left\{y_{i}, t_{i}\right\}$, such that the values of the model posterior probabilities $P(M / U)$ that ideally explains the data needed.

Ref. [8] propounded a general model for data, $f\left(t_{i}\right)$ to be

$$
y_{i}=f\left(t_{i}\right)+\varepsilon\left(t_{i}\right)
$$

such that the white noise $\varepsilon\left(t_{i}\right) \approx \operatorname{Gaussian}\left(0, \sigma^{2}\right) \forall, i=1, \mathrm{~L}, n$

Also, [9] specified out a Sinusoidal model to be

$$
f(t)=A \cos (w t)+B \sin (w t)
$$

For parameters $\theta=\{A, B, \omega\}, " A \& B "$ are the time axes while " $\omega "$ is the periodogram; for a typical noise model with zero mean Gaussian referred to Joint Conditional Distribution.

$$
P(U / \theta, M)=\frac{1}{\sigma_{i} \sqrt{2 \pi}} e^{-\left(\frac{y_{i}-f\left(t_{i}\right)}{2 \sigma_{i}}\right)^{2}}-\infty<y_{i}<\infty
$$

For the model to be stationary, then " $\sigma_{i}$ "will be replaced by a singular scalar of " $\sigma$ "

$$
P(U / \theta, M)=\frac{1}{\sigma \sqrt{2 \pi}} e^{-\left(\frac{y_{i}-f\left(t_{i}\right)}{2 \sigma}\right)^{2}}
$$

The total likelihood equals,

$$
P(U / \theta, M)=\prod_{i=1}^{n} \frac{1}{\sigma_{i} \sqrt{2 \pi}} e^{-\left(\frac{y_{i}-f\left(t_{i}\right)}{2 \sigma_{i}}\right)^{2}}
$$




$$
=\left(\frac{1}{\sigma \sqrt{2 \pi}}\right)^{n} e^{-\frac{1}{2 \sigma} \sum_{i=1}^{n}\left(y_{i}-f\left(t_{i}\right)\right)^{2}}=\sigma^{-n}\left(\frac{1}{\sqrt{2 \pi}}\right)^{n} e^{-\frac{1}{2 \sigma} \sum_{i=1}^{n}\left(y_{i}-f\left(t_{i}\right)\right)^{2}}
$$

Factoring out the constant that is proportional to the kernel density

$$
P(U / \theta, M) \infty \sigma^{-n} e^{\left(-\frac{R}{2 \sigma^{2}}\right)}, \text { where } R=\sum_{i=1}^{n}\left(y_{i}-f\left(t_{i}\right)\right)^{2}
$$

So expanding $\mathrm{R}$,

$$
\begin{gathered}
R=\sum_{i} y_{i}^{2}+\sum_{i} f\left(t_{i}\right)^{2}+2 \sum_{i} y_{i} f\left(t_{i}\right)=\sum_{i} y_{i}^{2}+\sum_{i} f\left(t_{i}\right)^{2}+2[A k(\omega)+B p(\omega)] \\
\text { for }, \quad k(\omega)=\sum_{i} y_{i} \cos \left(w t_{i}\right) \\
p(\omega)=\sum_{i} y_{i} \sin \left(w t_{i}\right) \quad \text { from eqn }(i i), f\left(t_{i}\right)=A \cos \left(w t_{i}\right)+B \sin \left(w t_{i}\right)
\end{gathered}
$$

So, $\sum_{i} f\left(t_{i}\right)=A^{2} \sum_{i} \cos ^{2}\left(\omega t_{i}\right)+B^{2} \sum_{i} \sin ^{2}\left(\omega t_{i}\right)+2 A B \sum_{i} \cos \left(\omega t_{i}\right) \sin \left(\omega t_{i}\right)$

But from trigonometry approximation which also coincide with [10]

When $i>1$,

$$
\begin{gathered}
\sum_{i} \sin ^{2}\left(\omega t_{i}\right)=\frac{n}{2}+\frac{1}{2} \sum_{i} \sin \left(\omega t_{i}\right) \cong \frac{n}{2} \\
\sum_{i} \cos ^{2}\left(\omega t_{i}\right)=\frac{n}{2}+\frac{1}{2} \sum_{i} \cos \left(\omega t_{i}\right) \cong \frac{n}{2} \\
\sum_{i} \cos \left(\omega t_{i}\right) \sin \left(\omega t_{i}\right)=\frac{1}{2} \sum_{i} \sin \left(2 \omega t_{i}\right)<\frac{n}{2},
\end{gathered}
$$

such that eqn (6) equals

$$
R=\sum_{i} y_{i}^{2}+\frac{n}{2}\left(A^{2}+B^{2}\right)-2[A k(\omega)+B p(\omega)]
$$


The exponentiated term $e^{\left(-\frac{R}{2 \sigma^{2}}\right)}$ in eqn (4) equals

$$
e^{\left(-\frac{R}{2 \sigma^{2}}\right)}=e^{\left(-\frac{\sum y_{i}^{2}}{2 \sigma^{2}}\right)} e^{\left(-\frac{n A^{2}}{4 \sigma^{2}}+\frac{k(\omega) A}{\sigma^{2}}\right)} e^{\left(-\frac{n B^{2}}{4 \sigma^{2}}+\frac{k(\omega) B}{\sigma^{2}}\right)}
$$

So, to get the Marginalized Posterior Probability Density Function over the frequency " $\omega "$

$$
P(\omega, \sigma / U, M)=\iint e^{\left(-\frac{R}{2 \sigma^{2}}\right)} \times \text { Prior } \partial A \partial B
$$

For simplicity, Uniform Prior (Improper prior) will be adopted because of its stretches from $-\infty$ to $+\infty$ in order to be able to integrate the function as it drops to zero as the magnitude of the amplitude increases.

$$
\text { So, } P(\omega, \sigma / U, M)=\iint e^{\left(-\frac{R}{2 \sigma^{2}}\right)} \partial A \partial B
$$

But recall from standard integral

$$
\begin{aligned}
\int_{-\infty}^{\infty} e^{\left(-c x^{2}-d x\right)} \partial x=\sqrt{\frac{\pi}{c}} e^{\left(\frac{d^{2}}{4 c}\right)} i f f c>0 \\
P(\omega, \sigma / U, M)=e^{\left(-\frac{\sum y_{i}^{2}}{2 \sigma^{2}}\right)} \int_{-\infty}^{\infty} e^{\left(-\frac{n A^{2}}{4 \sigma^{2}}+\frac{k(\omega) A}{\sigma^{2}}\right)} \partial A \int_{-\infty}^{\infty} e^{\left(-\frac{n B^{2}}{4 \sigma^{2}}+\frac{p(\omega) B}{\sigma^{2}}\right)} \partial B \\
=\left(-\frac{\sum y_{i}^{2}}{2 \sigma^{2}}\right) \\
2 \sigma \sqrt{\frac{\pi}{n}} e^{\left(\frac{k(\omega)^{2}}{n \sigma^{2}}\right)} 2 \sigma \sqrt{\frac{\pi}{n}} e^{\left(\frac{p(\omega)^{2}}{n \sigma^{2}}\right)}
\end{aligned}
$$




$$
\begin{gathered}
=\frac{\pi}{n} e^{\left(-\frac{\sum y_{i}^{2}}{2 \sigma^{2}}\right)} 4 \sigma^{2} e^{\left(\frac{k(\omega)^{2}+p(\omega)^{2}}{n \sigma^{2}}\right)} \\
=\frac{4 \pi}{n} \sigma^{2-n} e^{\left(-\frac{\sum y_{i}^{2}}{2 \sigma^{2}}+\frac{k(\omega)^{2}+p(\omega)^{2}}{n \sigma^{2}}\right)} \\
=P(\omega, \sigma / U, M) \infty \sigma^{2-n} e^{\left(-\frac{\sum y_{i}^{2}}{2 \sigma^{2}}+\frac{k(\omega)^{2}+p(\omega)^{2}}{n \sigma^{2}}\right)}
\end{gathered}
$$

Absorbing " $\sigma^{2} "$ in the first term in the exponent term into the proportionality constant, gives the posterior over " $\omega$ " to be the BAYESIAN PERIODOGRAM,

$$
P(\omega, \sigma / U, M) \infty e^{\left(\frac{W(\omega)}{\sigma^{2}}\right)}
$$

Where

$$
W(\omega)=\frac{1}{n}\left[k(\omega)^{2}+p(\omega)^{2}\right]=\frac{1}{n}\left|\sum_{i=1}^{i=n} y_{i} e^{-i \omega t n}\right|^{2}
$$

i.e $P(\omega, \sigma / U, M)$ is the BAYESIAN PERIODOGRAM FOR KNOWN NOISE.

If $\sigma$ is unknown, then a one of the non-informative priors will be used to multiply eqn (8). So, Jeffrey's prior of $p(\sigma)=\frac{1}{\sigma}$ will be multiply by eqn (8)

$$
P(\omega, \sigma / U, M)=\int_{0}^{\infty} \sigma^{1-n} e^{\left(-\frac{\sum y_{i}^{2}}{2 \sigma^{2}}+\frac{k(\omega)^{2}+p(\omega)^{2}}{n \sigma^{2}}\right)} \partial \sigma^{2}
$$




$$
\int_{0}^{\infty} \sigma^{1-n} e^{(-b)} \partial \sigma^{2}
$$

Where,

$$
b=\frac{\sum y_{i}^{2}}{2}-W(\omega)
$$

Recall from standard integral that

$$
\begin{gathered}
\int_{0}^{\infty} x^{f-3} e^{\left(-c x^{2}\right)} \partial x=\frac{1}{2} \Gamma\left(\frac{f-2}{2}\right) c^{\left(\frac{2-f}{2}\right)} \\
\text { So, } P(\omega, \sigma / U, M)=\frac{1}{2} \Gamma\left(\frac{n-2}{2}\right)\left(\frac{\sum y_{i}^{2}}{2}-W(\omega)\right)^{\left(\frac{2-n}{2}\right)}
\end{gathered}
$$

Absorbing terms not involving "W( $\omega)$ " into proportionality constant

$$
P(\omega, \sigma / U, M)=\infty\left(\frac{\sum y_{i}^{2}}{2}-W(\omega)\right)^{\left(\frac{2-n}{2}\right)} \quad \forall i>2
$$

\section{Eqn (IX) is the BAYESIAN PERIODOGRAM FOR UNKNOWN NOISE}

\section{Experimental Work}

The data used in this research was the readings rate of heartbeats of newly born babies in state owned hospital in Lagos state, Nigeria. These rates of heartbeat variability were recorded in two different timeframe (hours); rates of heartbeat an hour after birth and rates of heartbeat two hours after birth. The readings recorded were for one thousand eight hundred (1800) babies in the second half of 2016.

Fig.1 (a) and (b) shows the rates of heartbeat variability (the data) in black fitted into the sinusoidal model in blues. It was deduced that the impulse rates (signals) among the babies after an hour were widely unclosed compared to pulse rates that are more closely after two hours. In other words, the rates are considered to have been reduced when taking readings after two hours, which give rise to a more clustered rate in the second diagram.

Fig.2 (a) and (b) shows the Schuster periodogram (Fourier power spectral spectrum) and the Bayesian periodograms for both when the white noise (Sigma) is known and unknown after an hour of birth Fig.2 (a) and after two hours of birth Fig.2 (b). The periodograms for the unknown standard deviation for both the heartbeat frequencies variability exactly an hour and exactly after two hours seem to be more taper peak as other narrow bell-shape which suggested an approximate and alternative frequency to other no frills periodograms. 


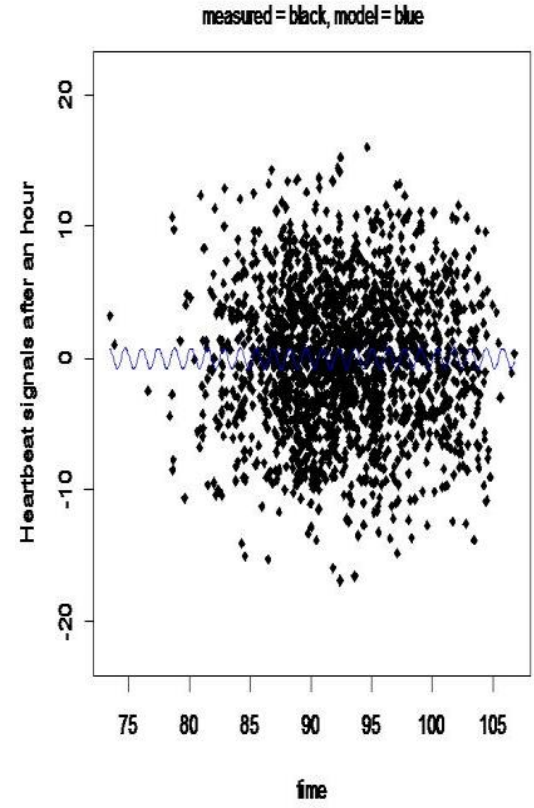

(a)

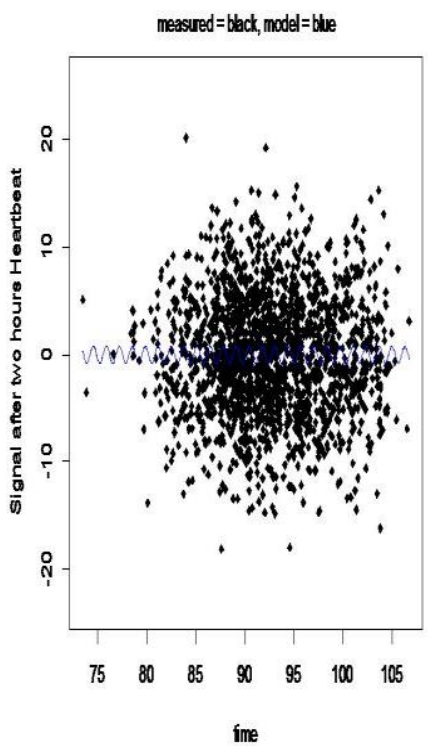

(b)

Fig.1. (a) The Heartbeat Signals After an Hour; (b) The Heartbeat Signals after an Two Hours
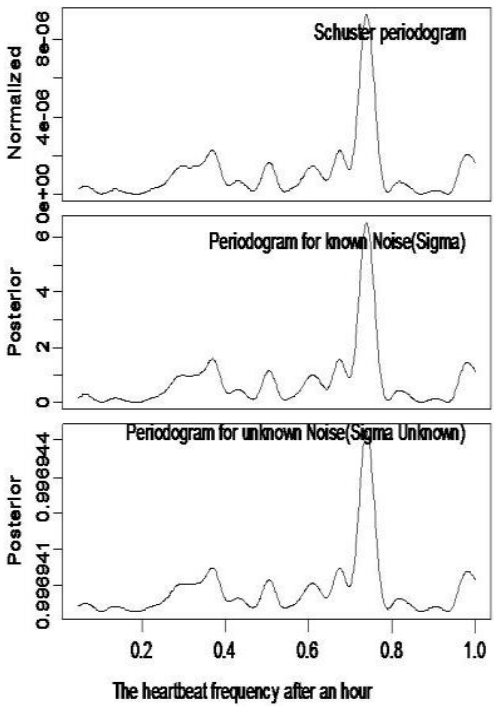

(a)
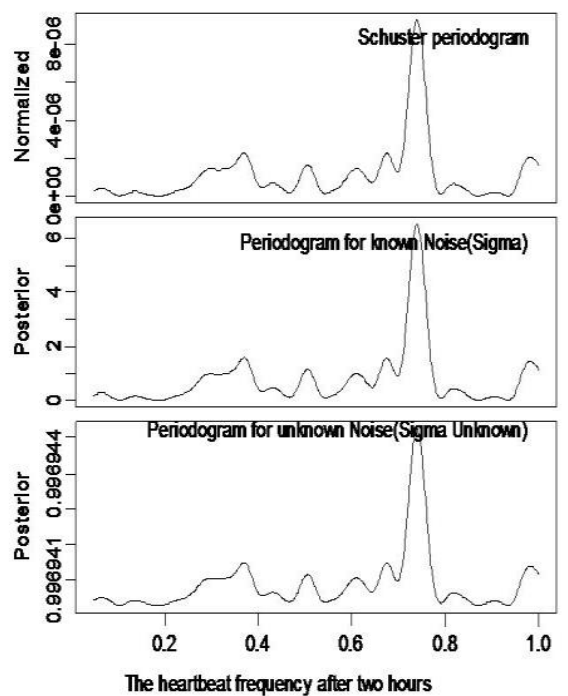

(b)

Fig.2. (a) The Periodogram of an Hour Rate; (b) The Periodogram of Two Hours Rate 

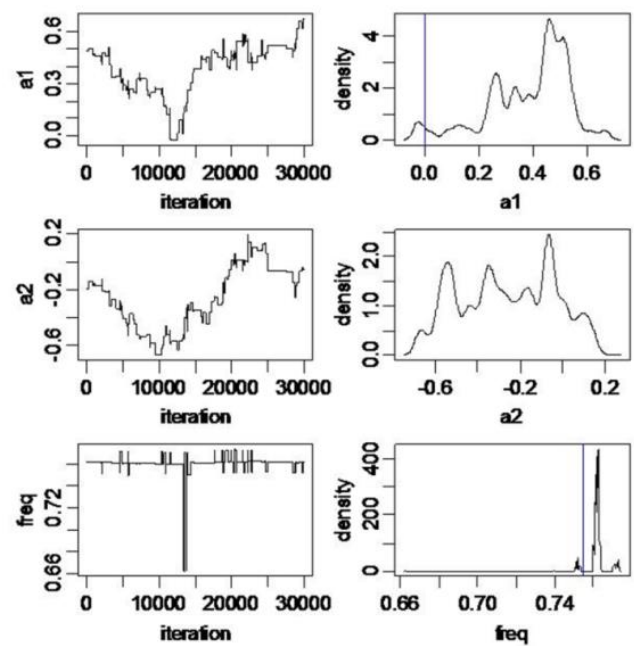

(a)
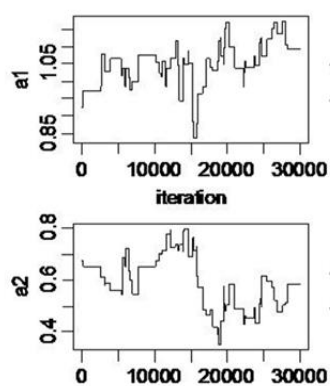

iteration
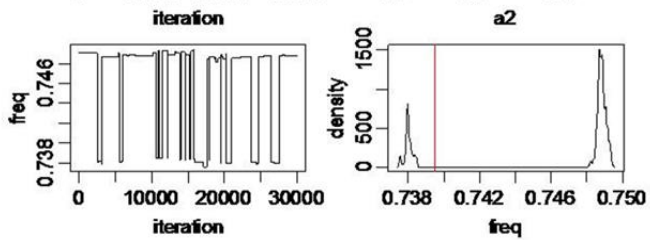

(b)

Fig.3. (a) Posterior Probability Density Function Sampling after an Hour' Rate; (b) Posterior Probability Density Function Sampling after Two Hours' Rate

The rectangular sections of the above fig.3 (a) and (b) are of two sides; the left and the right panels' columns for the captivity for the three parameters (the time axes and the periodograms) $\left\{A_{i}, B_{i}, \omega_{i}\right\}$ for $i=1,2$ such that the left panels showed the iteration of the parameters from the sinusoidal model through Markov Chain Monte Carlo (MCMC) log-likelihoods density estimation while the vertical blue and red lines in the right side panels for fig.3 (a) and (b) indicated the posterior parameters for the sinusoidal models for the rates after an hour and two hours' heartbeat. That is, $\left(A_{1}, B_{1}, \omega_{1}\right)=(0.0000000,0.7853982,0.7395200)$ $\left(A_{2}, B_{2}, \omega_{2}\right)=(0.0000000,0.7853982,0.7548969)$

Table 1. Posteriors' Parameter, Priors' Parameters and Log-likelihoods for the Hours

\begin{tabular}{|l|l|l|l|}
\hline \multicolumn{4}{|c|}{ An Hour Rates of Heartbeat } \\
\hline & $\begin{array}{l}\text { True } \\
\text { Parameter(Posterior) }\end{array}$ & Prior & $\log$ (Prior) \\
\hline$A_{1}$ & 0.00000 & 0.3989 & -0.3990 \\
\hline$B_{1}$ & 0.7854 & 0.2930 & -0.5330 \\
\hline$\omega_{1}$ & 0.7395 & 0.4631 & -0.3342 \\
\hline & & & -1.2663 \\
\hline
\end{tabular}

Log-likelihood of the sinusoidal model

$=-3534.019 ;$ PosMAP $=22234$

\begin{tabular}{|l|l|l|l|}
\hline \multicolumn{5}{|c|}{ Two Hours Rates of Heartbeat } \\
\hline$A_{2}$ & 0.0000 & 0.3989 & -0.3991 \\
\hline$B_{2}$ & 0.7854 & 0.2931 & -0.5330 \\
\hline$\omega_{2}$ & 0.7549 & 0.4608 & -0.3365 \\
\hline \multicolumn{3}{|c|}{} \\
$\begin{array}{l}\text { Log-likelihood of the sinusoidal model } \\
=-3632.588 ; \text { PosMAP }=20680\end{array}$ & -1.268576 \\
\hline
\end{tabular}

From Table .1 above, it can be deduced that the periodogram $\omega_{2}(0.7549)$ exactly after two hours of heartbeat rate was greater than of periodogram $\omega_{1}(0.7395)$ exactly after two hours of heartbeat rate of the 
sinusoidal model alluded and insinuated that the rates of heartbeat (signals) moderates and stabilizes the pulse among the babies. Also, the performance of the periodogram extracted from the sinusoidal model can be emphasized via the log-likelihood and the Posterior Maximum A Posteriori (PosMAP). According to [11], Bayesian models with equal number of parameter(s) can be compared via their log-likelihood and Bayesian maximum likelihood e:g PosMAP. The log-likelihood, PosMAP and sum of priors of the sinusoidal model after an hour and two hours rate of heartbeats $(-3534.019,22234,-1.2663)$ and $(=-3632.588,20680,-1.268576)$ respectively confirmed the stability in the rate of heartbeats among babies after two hours of birth, since it has already been established by that the model(s) with the most negative or minimum likelihood value.

Table 2. True means and Maximum a Posteriori Means of the Estimates

\begin{tabular}{|c|c|c|c|c|c|c|c|}
\hline \multicolumn{4}{|c|}{ An Hour Rates of Heartbeat } & \multicolumn{4}{|c|}{ Two Hours Rates of Heartbeat } \\
\hline & $\begin{array}{l}\text { True } \\
\text { Means }\end{array}$ & $\begin{array}{l}\text { Maximum A } \\
\text { Posteriori(MAP) } \\
\text { mean estimates }\end{array}$ & $\begin{array}{l}\text { Standrad Error } \\
\text { of estimates }\end{array}$ & & True Means & $\begin{array}{l}\text { Maximum A } \\
\text { Posteriori(MAP) } \\
\text { mean estimates }\end{array}$ & $\begin{array}{l}\text { Standrad } \\
\text { Error of } \\
\text { estimates }\end{array}$ \\
\hline$A_{1}$ & 0.7452885 & 0.6910084 & $2.083333 \mathrm{e}-06$ & $A_{2}$ & 0.3855557 & 0.54666803 & $2.083333 \mathrm{e}-06$ \\
\hline$B_{1}$ & 0.7432324 & 0.7528725 & $2.083333 \mathrm{e}-06$ & $B_{2}$ & 0.2614342 & 0.0158482 & $2.083333 \mathrm{e}-06$ \\
\hline$\omega_{1}$ & 0.7383294 & 0.7383849 & $8.333333 \mathrm{e}-06$ & $\omega_{2}$ & 0.7618441 & 0.76273958 & $8.333333 \mathrm{e}-06$ \\
\hline
\end{tabular}

Table II. Shows the True mean and MAP mean of estimates $\left\{A_{i}, B_{i}, \omega_{i}\right\}$ for $i=1,2$ estimated vie the Maximum Likelihood Estimation (MLE) and MCMC log-likelihood density estimation. In collaboration with fig.2, the MAP mean estimates are approximately equal to the True mean but not approximately equal to, in other not to be affected by over-parameterization and re-parameterization characterized by MLE [12]

\section{Conclusion}

One advantage of the choices of Bayesian inference has been safeguarding against over-fitting by integrating over model parameters (that is catered for problem of over-parameterization in one iteration) via MCMC exact uses of estimation with respect to sample size unlike the Schuster method that relied on asymptotic theory adopted by approximation of estimation, has seen in the values of the Maximum A Posteriori(MAP) mean estimates not exceeded the True Mean values because of over-parameterization and re-parameterization associated the MLE technique in estimation of parameters via Schuster method. In conclusion, the Bayesian approach seems to be clear-cut alternative in estimating the parameters especially the periodogram associated with the sinusoidal model when re-parameterization is not an option.

\section{References}

[1] Bailer-Jones C.A.L. Bayesian time series analysis and stochastic processes. Max Planck Institute for Astronomy, Heidelberg (2013), 1-31.

[2] Alexandrov, T. A. Method of trend extraction using singular spectrum analysis. Statistical Journal. 7(1), (2009), 1-22. doi: arXiv: 0804.33667v3

[3] Choudhuri, N., Ghosal, S., Roy, A. Bayesian estimation of the spectral density of a time series. Journal of the American Statistical Association. 99 (468), (2004), 1050-1059.

[4] Bhattacharya, B., Saha, B. Analysis of signaling time of community model, International Journal of Mathematical Sciences and Computing. 2(2), (2015), 8-21. doi:10.5815/ijmsc.2015.02.02. 
[5] Roy, K., Shelton, J., Esterline, A. A brief survey on multispectral iris recognition. International Journal of Applied Pattern Recognition. 3(4), (2016), 2049-8888. doi:.org/10.1504/IJAPR.2016.082235

[6] Wu, L., Ying, X., Wu, X. Examining spectral space of complex networks with positive and negative links. International Journal of Social Network Mining. 1(1) (2012), 91-111.

[7] Dias, C.N., Maurice, A., Prata, J.N. A refinement of the Robertson-Schrodinger uncertainty principle and a Hirschman-Shannon inequality for Wigner distributions. Journal of Fourier analysis Application. 2(3) (201), 1-32.

[8] Pantazis, Y., Oliver, R., Stylianous, Y. Iterative estimation of sinusoidal signal parameters. IEEE Signal Processing Letters. (2010).

[9] Prasad, A., Kundu, D., Mitra, A. Sequential estimation of the parameters of sum of sinusoidal model. Journal of Statistical planning and Inference. 3(38), (2008), 1297-1313.

[10] Bretthorst G.L. Bayesian spectrum analysis and parameter estimation. Lecture notes in statistics, Springer: (1998).

[11] Carson. C Chow. Bayesian Model Comparison. Scientific clearing house (2010):. https://sciencehouse.wordpress.com/2010/08/06/bayesian-model-comparison.

[12] Statisticat, LLC. Laplaces Demon: Complete Environment for Bayesian Inference (2015): Rpackage version 15.03.19,https://web.archive.org/web/20141224051720/http://www.bayesian-inference.com/index

\section{Authors' Profiles}

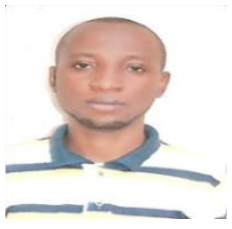

R.O. Olanrewaju is a Course Facilitator for the Distance Learning Centre (DLC), University of Ibadan on Statistical courses. He holds a Master of Science (Proceed to Ph.D. classification); Bachelor of Science (First Class honour) and Professional Diploma in Statistics (PDS) (Distinction) Statistics of the University of Ibadan. He is a member of the Nigeria Statistical Society (NSS). To his credit are some reputable articles and conference papers.

\section{APPENDIX A}

\section{A.1. THE PLOT OF RATES OF HEARTBEAT EXACTLY AFTER AN HOUR ONE}

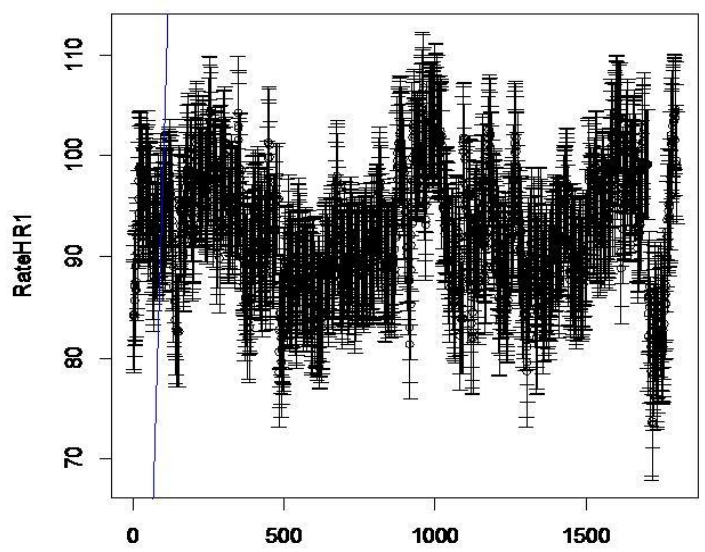




\section{A.2. THE PLOT OF RATES OF HEARTBEAT EXACTLY AFTER TWO HOURS BIRTH}

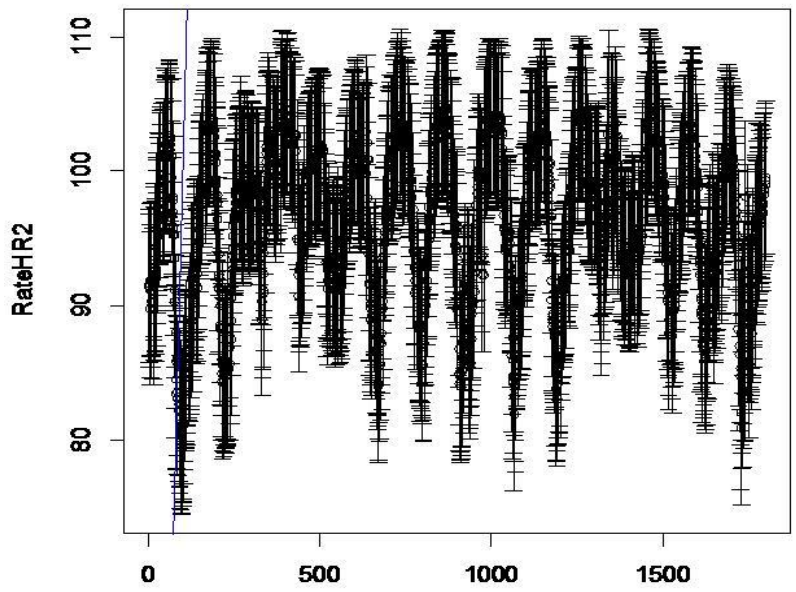

How to cite this paper: Olanrewaju Rasaki Olawale,"Bayesian Approach: An Alternative to Periodogram and Time Axes Estimation for Known and Unknown White Noise", International Journal of Mathematical Sciences and Computing(IJMSC), Vol.4, No.2, pp.22-33, 2018.DOI: 10.5815/ijmsc.2018.02.03 